\title{
Inorganic Islands on a Highly Stretchable Polyimide Substrate
}

\section{Citation}

Sun, Jeong-Yun, Nanshu Lu, Juil Yoon, Kyu-Hwan Oh, Zhigang Suo, and Joost J. Vlassak. 2009. Inorganic islands on a highly stretchable polyimide substrate. Journal of Materials Research 24(11): 3338-3342.

\section{Published Version}

doi:10.1557/JMR.2009.0417

\section{Permanent link}

http://nrs.harvard.edu/urn-3:HUL.InstRepos:4263768

\section{Terms of Use}

This article was downloaded from Harvard University's DASH repository, and is made available under the terms and conditions applicable to Open Access Policy Articles, as set forth at http:// nrs.harvard.edu/urn-3:HUL.InstRepos:dash.current.terms-of-use\#OAP

\section{Share Your Story}

The Harvard community has made this article openly available.

Please share how this access benefits you. Submit a story.

Accessibility 


\title{
Inorganic islands on a highly stretchable polyimide substrate
}

\author{
Jeong-Yun Sun ${ }^{1,2}$, Nanshu Lu², Juil Yoon³, Kyu-Hwan Oh, \\ Zhigang $\mathrm{Suo}^{2}$, and Joost J.Vlassak² a \\ ${ }^{1}$ Department of Material Science and Engineering, Seoul National University, Seoul 151-742, \\ South Korea. \\ ${ }^{2}$ School of Engineering and Applied Sciences, Harvard University, Cambridge, MA 02138, \\ USA. \\ 3 Department of Mechanical Systems Engineering, Hansung University, Seoul 136-792, \\ South Korea.
}

\begin{abstract}
For a flexible electronic device integrating inorganic materials on a polymer substrate, the polymer can deform substantially, but the inorganic materials usually fracture at small strains. This paper describes an approach to make such a device highly stretchable. A polyimide substrate is first coated with a thin layer of an elastomer, on top of which $\mathrm{SiN}_{x}$ islands are fabricated. When the substrate is stretched to a large strain, the $\operatorname{SiN}_{x}$ islands remain intact. Calculations confirm that the elastomer reduces the strain in the $\mathrm{SiN}_{x}$ islands by orders of magnitude.
\end{abstract}

Keywords: $\mathrm{SiN}_{x}$ islands, polyimide substrates, fracture, stretchability

a email: vlassak@esag.deas.harvard.edu 


\section{I . INTRODUCTION}

Flexible electronic devices may experience one-time or repeated large deformation during manufacture or in service. ${ }^{1-7}$ Such a device often integrates a polymer substrate with diverse inorganic materials, such as semiconductors, metals and ceramics. While the polymer can deform substantially, the inorganic materials usually fracture at strains below $\sim 1 \% .{ }^{8}$ Consequently, the device is stretchable only when the polymer and the inorganic materials are suitably integrated. Examples include inorganic islands 5, 9-11 and buckled inorganic films ${ }^{12,13}$ on polymer substrates.

This paper focuses on inorganic islands on a polymer substrate. For example, islands of diamond-like carbon on an elastomeric (PDMS) substrate remain intact when the substrate is stretched beyond $25 \% .{ }^{9}$ Some applications may require substrates much stiffer than PDMS, but $\mathrm{SiN}_{x}$ islands on much stiffer polyimide (PI) substrates crack and debond when the substrates are stretched by only a few percent. ${ }^{14,15}$ These two examples illustrate a tradeoff between the stiffness of the substrate and the stretchability of the structure.

Here we demonstrate that, when a thin layer of PDMS is sandwiched between the polyimide substrate and the $\operatorname{SiN}_{x}$ islands, the substrate can be stretched beyond $20 \%$ without causing the islands to crack or debond. The PDMS interlayer behaves as a buffer, isolating the islands from most of the strain of the substrate. The resulting $\mathrm{SiN}_{x} / \mathrm{PDMS} / \mathrm{PI}$ structure is both stretchable and relatively stiff.

\section{ПI. EXPERIMENTAL}

We fabricated two sets of specimens, $\mathrm{SiN}_{x} / \mathrm{PI}$ and $\mathrm{SiN}_{x} / \mathrm{PDMS} / \mathrm{PI}$, using the lift-off technique illustrated in Fig. 1(a). The substrates for the $\operatorname{SiN}_{x} /$ PI specimens were $25 \mu \mathrm{m}$ thick Upilex-S (UBE Industries), while the substrates for the $\mathrm{SiN}_{x} / \mathrm{PDMS} / \mathrm{PI}$ specimens were 70 $\mu \mathrm{m}$ thick Kapton-E (DuPont). Compared to Upilex-S, Kapton-E has a slightly lower Young's 
modulus but a somewhat larger strain to failure. All the PI foils were ultrasonically cleaned with methanol and acetone, and then attached to a $3 \mathrm{~mm}$ thick $\mathrm{Al}$ plate using double-sided tape. These bonded samples were then pressed in vacuum for 24 hours to remove bubbles between the PI foil and the Al plate.

The PDMS (Sylgard 184, Dow Corning) was prepared by mixing a silicone gel and a crosslinker in a 10:1 ratio by weight. A $10 \mu \mathrm{m}$ thick PDMS layer was then spin-coated on top of the Kapton-E. The specimens were degassed in vacuum for $20 \mathrm{~min}$, and cured for 1 hour at $80^{\circ} \mathrm{C}$. The surface of the PDMS was made hydrophilic by treating it for 20 seconds with an $\mathrm{O}_{2}$ plasma in a Technics Series 220 micro-stripper using a power of $75 \mathrm{~W}$.

A $3.2 \mu \mathrm{m}$ thick positive photoresist (S1818, Microposit) was spin-coated on top of the Upilex-S and PDMS/Kapton-E. The coated samples were baked for 2 min at $115{ }^{\circ} \mathrm{C}$ and then exposed through a Cr mask using an MJB4 mask aligner (SUSS MicroTec). The samples were developed in MF-319 photoresist developer (Microposit), rinsed in DI water for $1 \mathrm{~min}$, and dried with $\mathrm{N}_{2}$ gas. The substrates were then cut into $7 \mathrm{~mm} \times 60 \mathrm{~mm}$ rectangular strips and $500 \mathrm{~nm} \operatorname{SiN}_{x}$ films were deposited by chemical vapor deposition (CVD) in a NEXX system, with a base pressure of $5^{\times 10^{-6}}$ Torr and a working pressure of $10 \mathrm{mTorr}$. The microwave power was $265 \mathrm{~W}$ and the substrate temperature was held constant at $22{ }^{\circ} \mathrm{C}$. The gas flow rates for $3 \% \mathrm{SiH}_{4}$ (balance Ar), $\mathrm{N}_{2}$, and Ar were 40, 5.8, and 20 sccm respectively. Finally, the $\operatorname{SiN}_{x}$ coatings were patterned by stripping the extra $\operatorname{SiN}_{x}$ along with the remaining photoresist in acetone. At the same time, the samples were detached from the $\mathrm{Al}$ plate. Islands of various sizes were used, $L=40 \mu \mathrm{m}, 80 \mu \mathrm{m}, 120 \mu \mathrm{m}$, or $200 \mu \mathrm{m}$. The island thickness $h=500 \mathrm{~nm}$ and island period $S / L=1.5$ were the same for all specimens.

The specimens were stretched in a screw-driven tensile device (Figs.1b, c). The specimens were mounted with a $5 \mathrm{~mm} \times 5 \mathrm{~mm}$ square array of islands at the center to ensure that the arrays were subjected to uniform tensile strain. To reduce sliding between the 
specimens and the grips, two polymer tubes were attached to the samples with epoxy glue as shown schematically in Fig. 1(b). All tensile tests were performed in-situ under an optical microscope with CCD camera. Applied strains were measured directly on the recorded micrographs.

\section{RESULTS AND DISCUSSIONS}

Micrographs of the deformed islands are shown in Fig. 2. Islands on all $\mathrm{SiN}_{x} / \mathrm{PI}$ samples rupture at small strains. For samples with an island size of $200 \mu \mathrm{m}$, for instance, cracks first appear perpendicular to the tensile direction after an applied strain of $0.5 \%$. As shown in Fig. 2(a), all islands are fractured at an elongation of $0.8 \%$. If we reduce the island size, this strain-to-fracture increases slightly. For example, $40 \mu \mathrm{m} \mathrm{SiN} x$ islands start to crack when the substrate is stretched $0.9 \%$. As shown in Fig. 2(b), all $40 \mu \mathrm{m}$ islands have either cracked or debonded at an elongation of $2.1 \%$.

By contrast, $\mathrm{SiN}_{x} / \mathrm{PDMS} / \mathrm{PI}$ samples can be stretched to much larger strains. For samples with an island size of $200 \mu \mathrm{m}$, no cracking or debonding is observed in the islands after the sample is stretched by $20 \%$, as shown in Fig. 2(c). Upon further stretching, islands start to wrinkle due to the compressive transverse strain. Eventually, cracks appear in the islands at an elongation of $\mathbf{2 4 . 7 \%}$, as shown in Fig. 2(d). Island rupture can be completely suppressed by decreasing island size. It was impossible to fracture islands smaller than 200 $\mu \mathrm{m}$ because the PI substrates ruptured at elongations of about $30 \%$. This pronounced effect of the PDMS interlayer is also shown in Fig. 3, which plots the accumulated fraction of cracked islands as a function of applied strain.

Using the commercial finite element code ABAQUS, we have established a $3 \mathrm{D}$ model of a unit cell of the periodic island lattice. Schematics of the top and side views of the model are shown in Fig. 4. Because of symmetry, only one quarter of the unit cell was modeled, as 
represented by the shaded area in Fig. 4(a). We modeled the islands, the interlayer and the substrate as homogeneous, isotropic linear elastic materials with Young's moduli $E_{\mathrm{SiN}}=200$ $\mathrm{GPa}, E_{\mathrm{PDMS}}=1 \mathrm{MPa}$, and $\mathrm{E}_{\mathrm{PI}}=9.2 \mathrm{GPa}$, as well as Poisson's ratios $v_{\mathrm{SiN}}=v_{\mathrm{PI}}=0.3$ and $\nu_{\mathrm{PDMS}}=0.48^{16-18}$. The assumption of linear elasticity is quite good for the PDMS over the range of deformations considered here. ${ }^{19}$ The behavior of the PI is not as linear as that of the PDMS, but the precise PI stress-strain relationship has virtually no effect on the strain distributions in the PDMS layer and the $\mathrm{SiN}_{\mathrm{x}}$ islands, because the PI is so much stiffer than the PDMS. A uniform displacement $u_{\text {appl }}$ was applied to the substrates in the $x_{1}$-direction, i.e., the applied strain was given by $\varepsilon_{\text {appl }}=2 u_{\text {appl }} / S$.

The maximum tensile strain in the island, $\varepsilon_{\max }$, occurs at the center of the island edge, as labeled in Fig. 4(a). Fig. 5 plots ratios of the maximum strain in the island to the applied strain $\varepsilon_{\text {appl }}$ obtained from the simulations as a function of the island size. The maximum strain drops substantially when PDMS is introduced between the islands and the substrates. The simulations also show that the smaller islands experience smaller strains, but the effect is much more pronounced if the PDMS is introduced.

Assume that islands fracture when $\varepsilon_{\max }$ reaches a critical value $\varepsilon_{\mathrm{c}}=0.6 \%$. If we define the strain to failure, $\varepsilon_{\mathrm{r}}$, as the applied strain at which $50 \%$ of the islands are cracked, then $\varepsilon_{\mathrm{c}} / \varepsilon_{\mathrm{r}}$ can be plotted for the experiments and directly compared with the simulation results. For $\mathrm{SiN}_{\mathrm{x}} / \mathrm{PI}$ specimens with various island sizes, the experimental and corresponding FEM results are of the same order of magnitude. Compared to $\mathrm{SiN}_{x} /$ PI specimens, strains in islands on $\mathrm{SiN}_{x} / \mathrm{PDMS} / \mathrm{PI}$ specimens are much smaller, which confirms our previous claim that the large stretchability of $\operatorname{SiN}_{x} /$ PDMS/PI specimens is due to the reduced strains in the islands. The assumption that all islands crack whenever $\varepsilon_{\max }=\varepsilon_{c}$, independent of island size or thickness, is admittedly somewhat simplistic. This criterion only provides the correct 
order of magnitude when the FEM simulations are compared with the experiments. We will perform a more careful analysis of crack nucleation taking flaw size distributions into consideration in a future paper.

The strain in the islands on the PDMS interlayer can also be calculated using a simple shear-lag model.20 The detailed derivation is provided in the appendix. According to this model,

$$
\frac{\varepsilon_{\text {max }}}{\varepsilon_{\text {appl }}}=1-\frac{1}{\cosh \left(\frac{L}{2 \Lambda}\right)},
$$

where

$$
\Lambda=\sqrt{\frac{E_{\mathrm{SiN}} H h}{\mu_{\mathrm{PDMS}}}} .
$$

Here $\mu$ is the shear modulus and $H$ is the thickness of the interlayer. Equation (1) is plotted as the dashed curve in Fig. 5. The prediction of the shear-lag model agrees well with the finite element calculation. The length scale $\Lambda$ measures the distance over which normal strain builds up in the islands. For the materials used in this study, its value is approximately $1720 \mu \mathrm{m}$, i.e., one order of magnitude larger than the island size $L$. Consequently, the maximum strain in the islands is very small. Islands that are much larger than $\Lambda$, on the other hand, experience significant strain when the substrate is stretched. Evidently, $\Lambda$ is an important design parameter that can be used to determine the minimum buffer layer thickness required to relax the strain in an island of a given size and thickness. For instance, if the thickness of the buffer layer is reduced by a factor of two, the critical island size for strain relaxation decreases with a factor of $\sqrt{ } \mathbf{2}$. It should be noted that the shear-lag model does not account for interactions between the islands. If the island spacing is too small, the model breaks down.

Finally, we also performed a 2D island/substrate debonding analysis using the same finite element method mentioned in an earlier work. ${ }^{21}$ We found that the energy release rate 
for debonding, another important failure mode, is also reduced by orders of magnitude if a soft interlayer is applied.

\section{CONCLUSIONS}

In summary, when a thin layer of PDMS is introduced between the $\operatorname{SiN}_{\mathrm{x}}$ islands and the polyimide substrate, the substrate can be stretched beyond $20 \%$ without causing the islands to crack or debond. The soft PDMS layer acts as a buffer, isolating the $\operatorname{SiN}_{\mathrm{x}}$ islands from most of the applied strain in the polyimide substrate. This mechanism is confirmed by finite element simulations and a shear-lag model. The shear-lag model introduces a length scale that determines the island size for which the strain isolation is effective.

\section{ACKNOWLEDGMENTS}

This work was supported by the National Science Foundation (NSF) under Grant CMS-0556169, and by the Materials Research Science and Engineering Center (MRSEC) at Harvard University. It was performed in part at the Center for Nanoscale Systems (CNS), a member of the National Nanotechnology Infrastructure Network (NNIN), which is supported by the National Science Foundation under NSF Award No. ECS-0335765. CNS is part of the Faculty of Arts and Sciences at Harvard University. J-YS and KHO are grateful for support from the Korea Science and Engineering Foundation (KOSEF) granted by the Korea government (MOST, No.R11-2005-065). JY acknowledges support from the Korea Research Foundation funded by the Korean Government (KRF-2009-0069545).

\section{Appendix: Derivations of the shear-lag model}

As depicted in Fig. 4(c), the strain transfer between the PI substrate and a $\mathrm{SiN}_{\mathrm{x}}$ island via the compliant PDMS layer can be described with a simple 2D shear-lag model. Island length, thickness, and PDMS thickness are denoted by $L, h$, and $H$ respectively. In the model, 
the PI substrate is subjected to a uniform displacement $u_{0}\left(x_{1}\right)=\varepsilon_{\text {appl }} x_{1}$; the displacement field inside the island is assumed to be uniform through its thickness and is denoted by $u\left(x_{1}\right)$. Assuming uniform shear deformation through the thickness of the PDMS layer, the shear stress in the PDMS layer can be written as

$$
\tau\left(x_{1}\right)=\mu_{\mathrm{PDMS}} \frac{u\left(x_{1}\right)-u_{0}\left(x_{1}\right)}{H} .
$$

The shear stress at the $\operatorname{SiN}_{\mathrm{x}} /$ PDMS interface causes the normal stress in the island to vary. A force balance for an infinitesimal segment of the island yields

$$
\tau\left(x_{1}\right)=h \frac{d \sigma_{11}}{d x_{1}}=E_{\mathrm{SiN}} h \frac{d^{2} u}{d x_{1}^{2}}
$$

Equating (A1) and (A2) results in a second order ordinary differential equation in $u\left(x_{1}\right)$ :

$$
\Lambda^{2} \frac{d^{2} u}{d x_{1}^{2}}=u\left(x_{1}\right)-\varepsilon_{\text {appl }} x_{1},
$$

where

$$
\Lambda=\sqrt{\frac{E_{\mathrm{SiN}} H h}{\mu_{\mathrm{PDMS}}}} .
$$

Two boundary conditions are needed to solve Eq. (A3):

$$
\begin{gathered}
u\left(x_{1}=0\right)=0, \\
\sigma_{11}\left(x_{1}=\frac{L}{2}\right)=\left.E_{\mathrm{SiN}} \frac{d u}{d x_{1}}\right|_{x_{1}=\frac{L}{2}}=0 .
\end{gathered}
$$

The solution to Eq. (A3) is then given by

$$
u\left(x_{1}\right)=\varepsilon_{a p p l}\left[x_{1}-\Lambda \frac{\sinh \left(\frac{x_{1}}{\Lambda}\right)}{\cosh \left(\frac{L}{2 \Lambda}\right)}\right] .
$$

The corresponding strain field in the island is 


$$
\varepsilon\left(x_{1}\right)=\frac{d u}{d x_{1}}=\varepsilon_{\text {app } 1}\left[1-\frac{\cosh \left(\frac{x_{1}}{\Lambda}\right)}{\cosh \left(\frac{L}{2 \Lambda}\right)}\right] .
$$

The maximum strain in the island occurs at the center of the island where $x_{1}=0$, i.e.,

$$
\frac{\varepsilon_{\max }}{\varepsilon_{\text {appl }}}=1-\frac{1}{\cosh \left(\frac{L}{2 \Lambda}\right)} .
$$




\section{References}

1. J. A. Rogers, Z. Bao, K. Baldwin, A. Dodabalapur, B. Crone, V. R. Raju, V. Kuck, H. Katz, K. Amundson, J. Ewing, and P. Drzaic: Paper-like electronic displays: Large-area rubber-stamped plastic sheets of electronics and microencapsulated electrophoretic inks. Proc. Natl. Acad. Sci. U. S. A. 98, 4835 (2001).

2. E. Bonderover and S. Wagner: A woven inverter circuit for e-textile applications. IEEE Electron Device Lett. 25, 295 (2004).

3. C. J. Brabec: Organic photovoltaics: technology and market. Sol. Energy Mater. Sol. Cells 83, 273 (2004).

4. E. Abad, S. Zampolli, S. Marco, A. Scorzoni, B. Mazzolai, A. Juarros, D. Gómez, I. Elmi, G. C. Cardinali, J. M. Gómez, F. Palacio, M. Cicioni, A. Mondini, T. Becker, I. Sayhan: Flexible tag microlab development- Gas sensors integration in RFID flexible tags for food logistic. Sens. Actuator B-Chem. 127, 2 (2007).

5. D.-H. Kim, Y. -S. Kim, J. Wu, Z. Liu, J. Song, H. -S. Kim, Y. Huang, K. -C. Hwang, and J. A. Rogers: Ultrathin silicon circuits with strain isolation layers and mesh layouts for high performance electronics on fabric, vinyl, leather and paper. Adv. Mater. 21, 1 (2009).

6. T. Someya, Y. Kato, T. Sekitani, S. Iba, Y. Noguchi, Y. Murase, H. Kawaguchi, and T. Sakurai: Conformable, flexible, large-area networks of pressure and thermal sensors with organic transistor active matrixes. Proc. Natl. Acad. Sci. U. S. A. 102, 12321 (2005).

7. H. C. Ko, M. P. Stoykovich, J. Song, V. Malyarchuk, W. M. Choi, C. -J. Yu, J. B. Geddes III, J. Xiao, S. Wang, Y. Huang, and J. A. Rogers: A hemispherical electronic eye camera based on compressible silicon optoelectronics. Nature 454, 748 (2008).

8. H.-J. Oh, Y. Isono, T. Namazu, Y. Saito, A. Yamaguchi: Influence of Gas Flow Ratio in PE-CVD Process on Mechanical Properties of Silicon Nitride Film. IEEJ Trans. Electr. Electron. Eng. 3, 281 (2008).

9. S. P. Lacour, S. Wagner, R. J. Narayan, T. Li, Z. Suo: Stiff subcircuit islands of diamondlike carbon for stretchable electronics. J. Appl. Phys. 100, 014913 (2006).

10. P. I. Hsu, R. Bhattacharya, H. Gleskova, M. Huang, Z. Xi, Z. Suo, S. Wagner, and J. C. Sturm: Thin-film transistor circuits on large-area spherical surfaces. Appl. Phys. Lett. 81, 1723 (2002)

11. R. Bhattacharya, S. Wagner, Y. -J. Tung, J. R. Esler, and M. Hack: Organic LED Pixel Array on a Dome. Proc. IEEE 93, 1273 (2005).

12. D. -Y. Khang, H. Jiang, Y. Huang, J. A. Rogers: A stretchable form of single-crystal silicon for high-performance electronics on rubber substrates. Science 311, 208 (2006)

13. D. -H. Kim, J. -H. Ahn, W. M. Choi, H. -S. Kim, T. -H. Kim, J. Song, Y. Y. Huang, Z. Liu, C. Lu, J. A. Rogers: Stretchable and foldable silicon integrated circuits. Science 320, 507 (2008). 
14. H. Gleskova, S. Wagner, and Z. Suo: Failure resistance of amorphous silicon transistors under extreme in-plane strain. Appl. Phys. Lett. 75, 3011 (1999).

15. R. Bhattacharya, A. Salomon, and S. Wagner: Fabricating metal interconnects for circuits on a spherical dome. J. Electrochem. Soc. 153, G259 (2006).

16. O. Tabata, K. Kawahata, S. Sugiyama, and I. Igarashi: Mechanical property measurements of thin films using load-deflection of composite rectangular membranes. Sensors and Actuators 2o, 135 (1989).

17. S. H. Yoo, C. Cohen, C. -Y. Hui: Mechanical and swelling properties of PDMS interpenetrating polymer networks. Polymer 47, 6226 (2006).

18. The data sheet of Upilex-S, UBE INDUSTRIES.LTD.

19. A. L. Larsen, K. Hansen, P. Sommer-Larsen, O. Hassager, A. Bach, S. Ndoni, M. Jorgensen: Elastic properties of nonstoichiometric reacted PDMS networks. Macromolecules, 36, 10063 (2003).

20. Z. C. Xia, J. W. Hutchinson: Crack patterns in thin films. J. Mech. Phys. Solids, 48, 1107 (2000).

21. N. Lu, J. Yoon, and Z. Suo: Delamination of stiff islands patterned on stretchable substrates. Int. J. Mater. Res. 98, 717 (2007). 


\section{Figure Captions}

Fig. 1. (a) Fabrication sequence showing two sets of polyimide-supported island arrays in cross section: $\mathrm{SiN}_{x} / \mathrm{PI}$ and $\mathrm{SiN}_{x} /$ PDMS/PI. (b) Schematics of the in-situ uniaxial tensile test. (c) A picture of our homemade screw-driven tensile tester.

Fig. 2. Samples stretched horizontally: (a) $\mathrm{SiN}_{x} / \mathrm{PI}, L=200 \mu \mathrm{m}, \varepsilon_{\text {appl }}=0.8 \%$, all islands have channel cracks. (b) $\mathrm{SiN}_{x} / \mathrm{PI}, L=40 \mu \mathrm{m}, \varepsilon_{\text {appl }}=2.1 \%$, most islands are cracked but a few are debonded. (c) $\operatorname{SiN}_{x} / \mathrm{PDMS} / \mathrm{PI}, L=200 \mu \mathrm{m}, \varepsilon_{\text {appl }}=19.6 \%$, all islands are intact. (d) $\mathrm{SiN}_{x} / \mathrm{PDMS} / \mathrm{PI}, L=200 \mu \mathrm{m}, \varepsilon_{\text {appl }}=24.7 \%$, islands are wrinkled and cracked.

Fig. 3. The accumulated fraction of cracked islands is plotted as a function of the applied strain.

Fig. 4. (a) Top view and (b) side view of the 3D finite element model. (c) Schematic of the displacements in the shear-lag model: The displacement inside the PI substrate is given by $u_{0}\left(x_{1}\right)=\varepsilon_{\text {appl }} x_{1}$; the unknown displacement inside the island is assumed to be uniform through the thickness and is denoted by $u\left(x_{1}\right)$. The shear strain in the PDMS layer is $\left(\mathrm{u}\left(\mathrm{x}_{1}\right)-\right.$ $\left.\mathrm{u}_{\mathrm{o}}\left(\mathrm{x}_{1}\right)\right) / \mathrm{H}$.

Fig. 5. Ratios of the maximum strain in the island to the applied strain obtained from $3 \mathrm{D}$ finite element simulations, experimental results, and calculations using a shear lag model. They all illustrate the fact that a soft interlayer can greatly reduce strains experienced by the islands. 
(a)

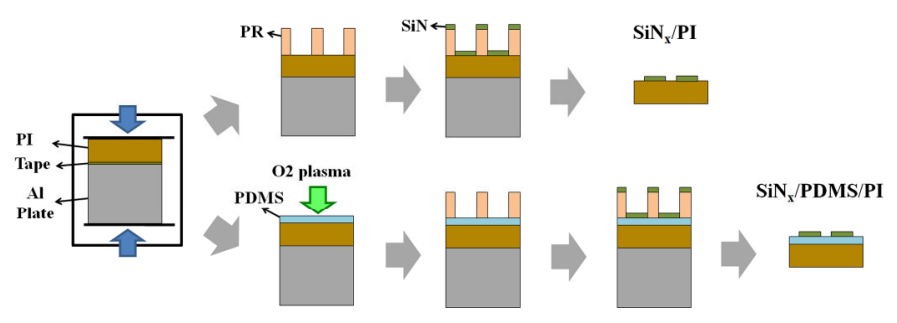

(b)

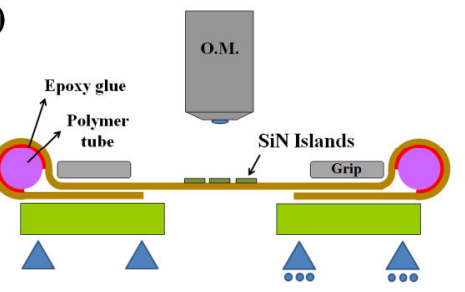

(c)

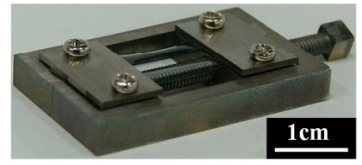

Figure 1 
(a)

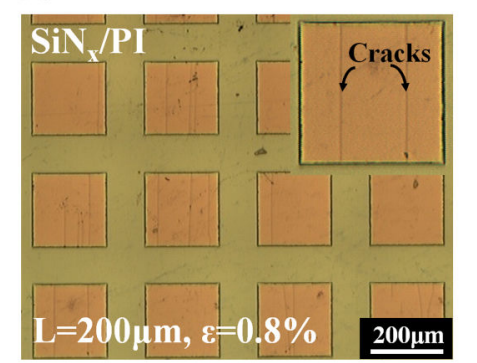

(c)

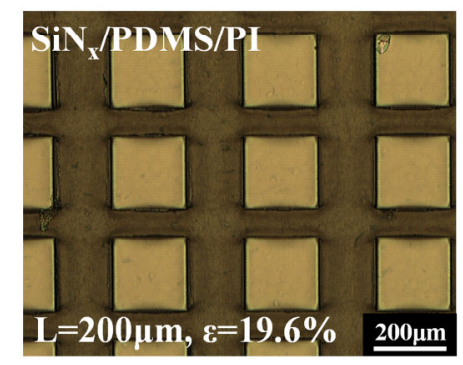

(b)

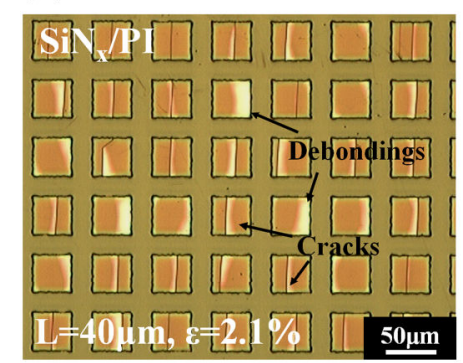

(d)

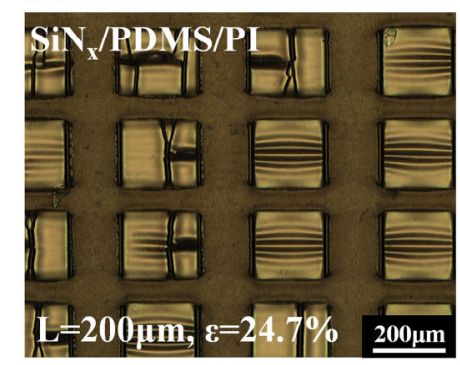

Figure 2 


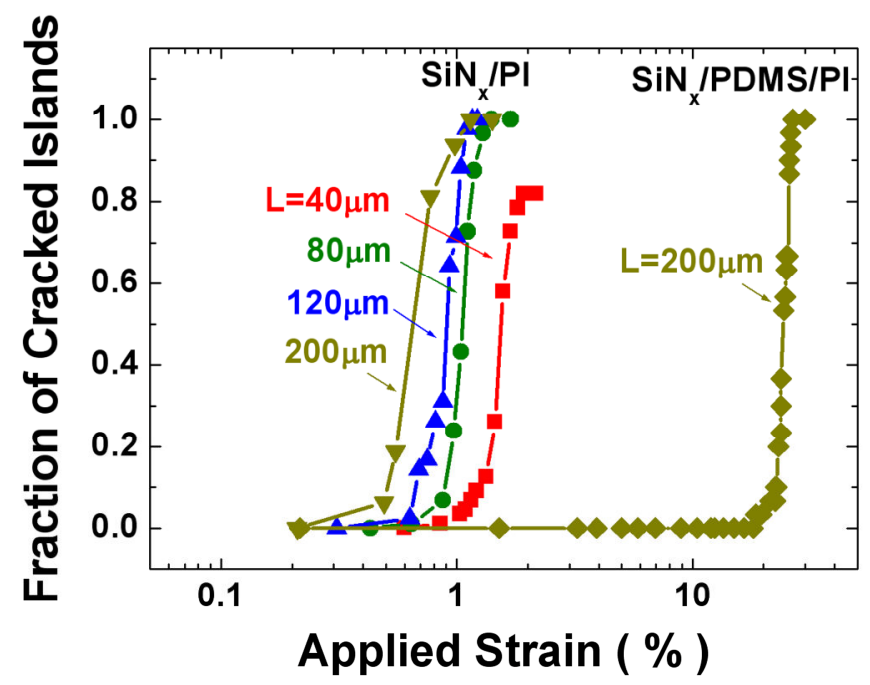

Figure 3 
(a)

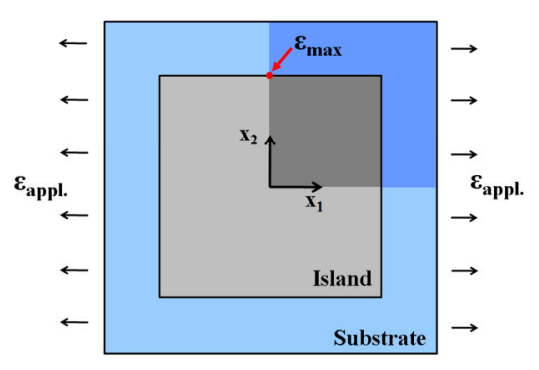

(b)

(c)
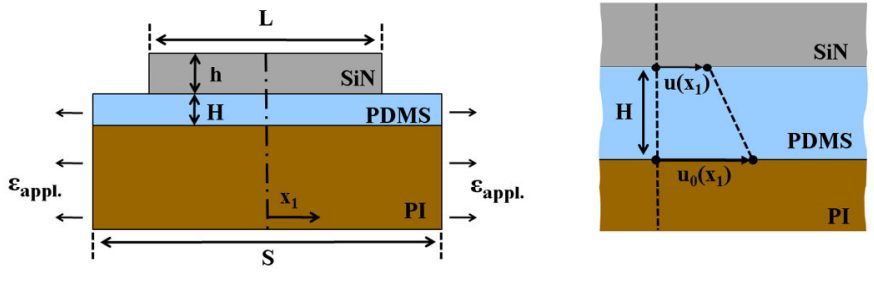

Figure 4 


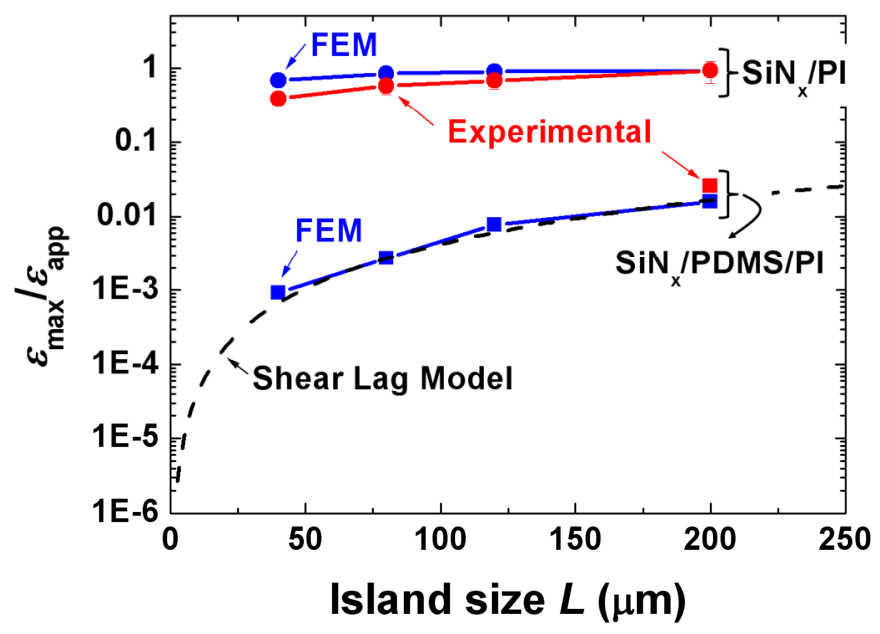

Figure 5 